\title{
Features of Medical Rehabilitation of Patients with Fractures of Tibia Older than 55 Years
}

\author{
Schurov Vladimir Alekseevich* \\ Researcher of the laboratory for correction of deformities of limb lengthening and RRC “WTO” them, Russia
}

Received: June 05, 2018; Published: June 13, 2018

*Corresponding author: Schurov Vladimir Alekseevich, doctor. Honey. Sciences, Professor, chief researcher of the laboratory for correction of deformities of limb lengthening and RRC “WTO” them. academician. G. A. Ilizarov. Russia, Kurgan, 640001, Koli Myagotina str. 95 . App. -49

\begin{abstract}
The goal is the analysis of the dynamics of micromotion of bone fragments and duration of treatment by Ilizarov patients older than 55 years with fractures of the Shin bones (220 people) in the conditions of outpatient treatment.

Method: Definition of micromotion of bone fragments was performed with a strain gauge assessment of changes in the distance between the spokes of the Ilizarov emerging from the tibia above and below the area of bone regenerate during the tests with stepwise increasing the axial load on the limb $10 \mathrm{kgs}$. The linear velocity of blood flow through the vessels in the area of bone regenerate along the anterior-abdominal surface of the tibia was determined by using an ultrasonic Doppler installation. Radiographs after treatment planimetrically determined the amount periosteal blood of callus.
\end{abstract}

Results: At transition of patients with injuries of a Shin on an outpatient mode of treatment practically in 2 times micro mobility of bone fragments and duration of the period of fixing increased. Indicators significantly increased in patients with increasing age to 30-55 years, and then tended to decrease. Compaction of bone regenerate during the fixation period contributed to the reduction of micro-mobility and normalization of blood flow rate in the regeneration zone. The reason for the reduction of treatment in older people we see in a gentler motor mode.

Keywords: PE: Preeclampsia; CO: Cardiac Output; CS: Caesarean Section; FC: Fluid Challenge; CI: Cardiac Index; MAP: Mean Arterial Pressure; SVI: Stroke Volume Index; FR: Fluid Responsiveness; SV: Stroke Volume

\section{Introduction}

The most important distinctive features of the treatment of patients with bone fractures by Ilizarov is the possibility of accurate reposition and reliable fixation of bone fragments, which allows to create optimal conditions for the implementation of reparative bone regeneration and for the restoration of locomotor activity of patients with functional loading of the injured limb. In vivo study of the axial deformation of the regenerate to an accuracy of $1 \mu \mathrm{m}$ is possible by the mutual convergence of the Ilizarov apparatus spokes emerging from the bone fragments under functional loading of the limb [1]. The use of circular fixation by Ilizarov in surgical treatment of limbs in comparison with fixation by rod monolateral devices that do not have the necessary rigidity of fixation of fragments is accompanied by a much smaller number of clinical errors and complications (regenerate deformation, dislocation in the joints) (5-8\% compared to $25-33 \%)$ [2,3]. The aim of this work was the comparative analysis of the influence of the rigidity of fixation of bone fragments at different stages of the compression osteosynthesis for the duration of the treatment period in patients older than 55 years.

\section{Material and methods}

The study included 64 patients aged 20 to 84 years $(42 \pm 1.7$ years) with closed Shin bone fractures treated on an outpatient basis after 2 weeks of hospital stay. In all patients, a sample with a stepwise increasing axial load on the limb with a force of $10 \mathrm{~kg}$ was carried out, while tensometrically determined the changes in the distance between the spokes of the Ilizarov apparatus emerging from the tibia above and below the bone regenerate zone, which allowed to determine the micro-mobility of bone fragments [1]. On radiographs after removal of the device, the diameter of the diaphysis of the maternal bone in the fracture zone, as well as the maximum diameter of the bone in the periosteal zone, the difference in diameters, and the length of the periosteal reaction were determined. The area of periosteal growth was estimated as half of the product of the height and length of the formation. 
Using ultrasonic Doppler unit "Angiodin-2KM" (Russia) was estimated linear systolic blood flow rate through the vessels in the area of bone regenerate, on the anterior surface of the tibia. Statistical processing of research results was carried out using the data analysis package "Microsoft Exell-2010. The average values of the indicators and the standard error $(\mathrm{M} \pm \mathrm{m})$ are given. In the analysis of the dynamics of changes in indicators, the methods of correlation and linear regression analysis (C denotation of the $\mathrm{R} 2$ determination index) were used. To assess the statistical significance of differences between the results of two independent samples, student t-test was used.

\section{Research result}

In the treatment of patients after osteosynthesis after 15 days of inpatient treatment for outpatient treatment, there was a period of increase in the micro-mobility of bone fragments due to an increase in the functional load on the injured limb. The duration of the fixation period increased from $59 \pm 1.4$ to $98 \pm 3.8$ days $(p \leq 0.001)$. A significant impact on the timing of fixing the fragments had an increase in the age of patients (Figure 1). The duration of the fixation period increased during the first 35 years of life (as the strength of the bones and, accordingly, the force of traumatic effects increased). After 55 years, there was a decrease in the time of fixation of bone fragments. In patients from 21 to 55 years $(37 \pm 2,9$ years $)$ the average duration of fixation was $115 \pm 11$, and in patients $62 \pm$ years $-81 \pm 12$ days.

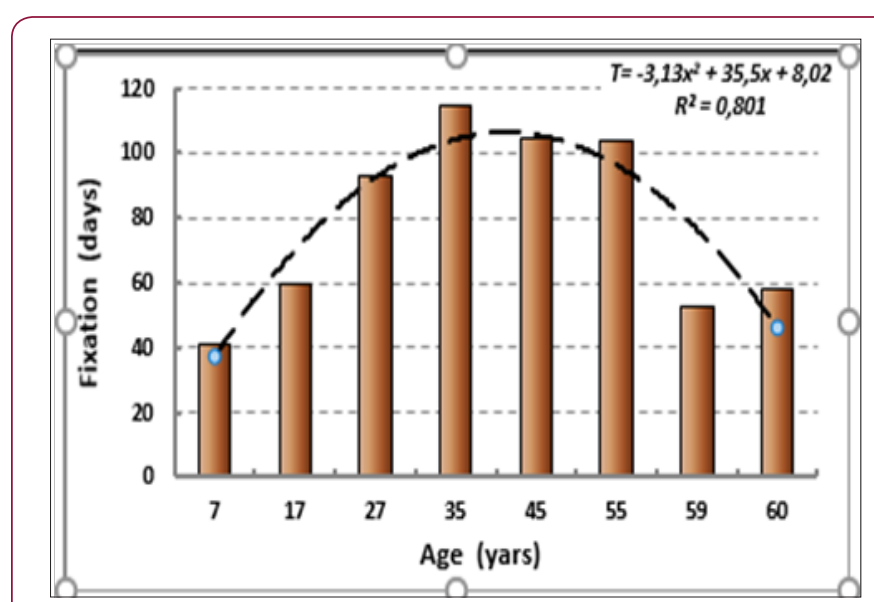

Figure 1: Age dynamics duration of the fixation period in the treatment of patients on an outpatient basis.

One of the reasons for the reduction of the duration of the fixation period in elderly patients could be a decrease after 55 years of micro-mobility of bone fragments, which increased with age in young patients (Figure 2). It turned out that the magnitude of the micro-mobility of bone fragments depends on the intensity of blood supply to the corn. As the micro-mobility increased to $20-60 \mu \mathrm{m}$, the blood flow rate increased for each $10 \mathrm{~kg}$ of load. However, with large values of micro-mobility, typical for patients during treatment in outpatient settings-steadily decreased (Figure 3). With the excessive force of compression and the lack of rigidity of bone fragments fixation is an increase in the periosteal monolaurate (Figure 4), it is a compensatory reaction, contributing to an increase in the cross-sectional area of the regenerate and reduce the specific pressure on it at rest and under functional loading of the limb, which is important to improve blood supply to the ends of the fragments [4]. The size of the periosteal regenerate increased in the treatment of patients in outpatient settings as the age increased to 55 years, and at an older age began to decrease (Figure 5).

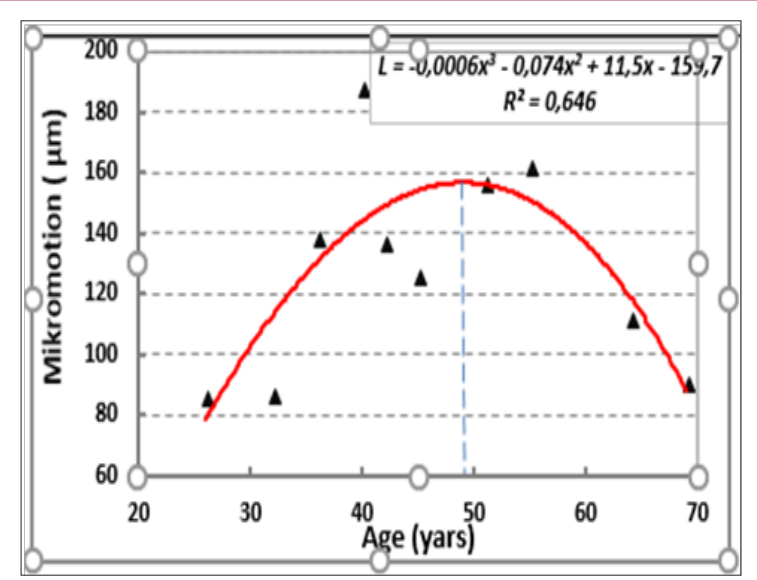

Figure 2: Age dynamics of micro-mobility of bone fragments under functional load on the limb.

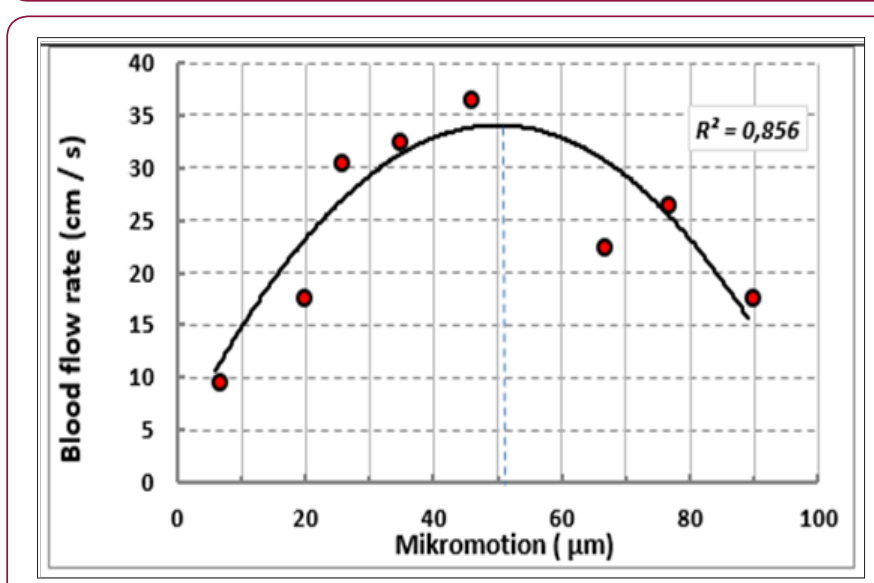

Figure 3: The relationship of micro-mobility of fragments in the elongation of the lower leg and the speed of blood flow through the vessels of bone regenerate.

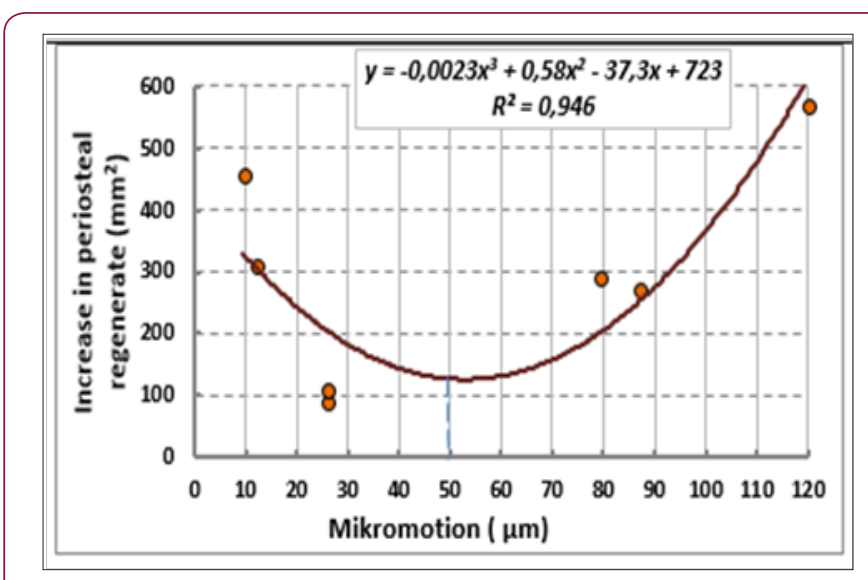

Figure 4: The relationship between the stiffness of bone fragments fixation and the size of periosteal regenerate. 


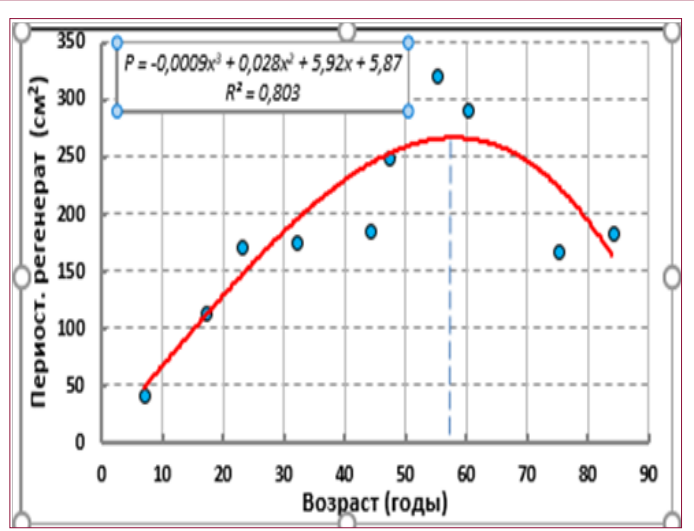

Figure 5: Age dynamics of the periosteal regenerate size in the treatment of patients.

\section{Discussion of Results}

The transfer of patients with Shin bone fractures to outpatient treatment mode helps to increase the "bed turnover", increase the surgical activity of traumatologists, creates conditions for increasing the availability of high-tech treatment for the population of the country's regions. However, in the treatment of Ilizarov significantly (almost 2 times) increased the time of fixation of the limb. The dependence of the treatment period on the age of patients was clearly manifested. It would seem that the most unfavorable conditions should be people of retirement age with limited mobility. However, it was found out that in the treatment in outpatient conditions in patients older than 55 years, the micro-mobility of bone fragments decreases less, the periosteal reaction of the bone is less pronounced, and the treatment period is relatively shorter. A possible cause of the observed phenomena in older patients with this form of treatment is relatively less reactivity of the body, motor activity and functional load on the damaged limb.

The need for functional loading of the limb to stimulate osteogenesis is not in doubt. Currently, even when using the locking plate structures provides the possibility of dynamic modification during loading of the limb [5]. If the micromotion increased up to $40-50 \mu \mathrm{m}$ increase the blood flow to the affected limb, which is favorable to reparative regeneration, with increase of the micromotion to $80-140 \mu \mathrm{m}$, and very slow compaction of callus and increase parietalnyh layers. This is a compensatory reaction to the reduction of fixation stiffness, since it helps to reduce the specific pressure on the regenerate during locomotion, that is, to increase a painless load on the limb, while excluding a violation of the blood supply to the bone marrow. It should also be noted such a positive phenomenon as the lack of increase in the duration of the period of disability with the extension of the period of fixation for more than 65 days. In cases of such lengthening of the fixation period, a compensatory reduction of the subsequent period of functional rehabilitation occurs.

\section{Conclusion}

In the treatment of fractures, the micro-mobility of bone fragments, which determines the duration of the fixation period, increased during the transition of patients to outpatient treatment. In patients with increasing age to 55 years, increases the micromobility of bone fragments, periosteal layers in the regenerate and the duration of the period of fixation. In patients older than 55 years in terms of age-related reduction of motor activity, the transition to outpatient treatment is not accompanied by such pronounced changes in the mobility of the fragments and in the terms of treatment.

\section{References}

1. Schurov VA (2014) Elastic compliance and blood flow to the distraction of regenerate. Russian journal of biomechanics18(4): 471-478.

2. Pouliquen JC (1991)La strategie: Traitement des inegalites de longueur des membresinferieurs et des sujets de petite taille chez l'enfant etl'adolescent: symp. Sous dir De JCatonRevChirOrthop77(Suppl2): 5354.

3. Pouliquen JC, Glorion Ch, Langlais J (1996) Allongement de membrespar la metode du callotasis. //Cahiers deenseignement de la SOFCOT n ${ }^{\circ} 58$. Paris: Expansion SientifiqueFrançaise pp. 293-302.

4. Schurov VA, Mesnard M (2018) Evalution of the biomechanical and functional maturity of the bone regenerate.Human Physiology4(1): 102107.

5. Laurence M, Frelman MAR, Swenson SAV (1999) Engineerung consideration in the internal fixation of fractures of the tibial shaft.JBone ItSurg51(4): 754-769.
This work is licensed under Creative Commons Attribution 4.0 License

Submission Link: https://biomedres.us/submit-manuscript.php

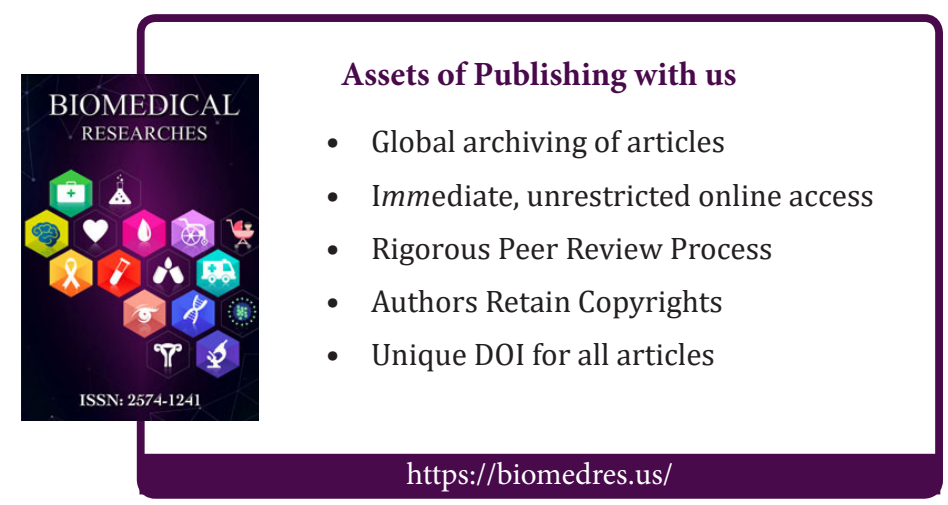

